\title{
Erratum to: \\ Enzyme-Free Dissociation of Neurospheres \\ by a Microfluidic Chip-Based Method
}

\section{Ching-Hui Lin, Hao-Chen Chang, Don-Ching Lee, Ing-Ming Chiu, and Chia-Hsien Hsu}

Methods in Molecular Biology (2016) 1516: 289-297

DOI 10.1007/7651_2016_348

(C) Springer Science+Business Media New York 2016

Published online: 05 April 2016

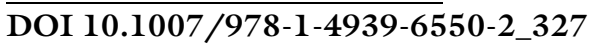

The publisher regrets that two authors were not mentioned in the chapter by mistake. The correct order of authors is provided below:

Ching-Hui Lin, Hao-Chen Chang, Don-Ching Lee, Ing-Ming Chiu, and Chia-Hsien Hsu 